\title{
Automedicação entre universitários da área da saúde no interior do Tocantins
}

A Agência Nacional de Vigilância Sanitária (ANVISA) conceitua a automedicação como uso indiscriminado de medicações sem a prescrição, orientação e acompanhamento de um profissional da área da saúde (médico ou dentista). O ato de se automedicar entre os acadêmicos da área da saúde é considerado bastante comum nas instituições de ensino superior. Tem como objetivo em identificar os fatores associados e a prevalência ligada à automedicação em estudantes de uma instituição de ensino superior da área da saúde no interior do Tocantins. Este estudo é uma pesquisa de campo, transversal com análise quantitativa, seleção de amostra por meio randomizado. A pesquisa foi realizada com os acadêmicos de Enfermagem, Biomedicina e Fisioterapia matriculados no Instituto Educacional Santa Catarina - IESC - Faculdade Guaraí - FAG, no Tocantins, através de um questionário virtual contendo 20 questões. A pesquisa é composta por 198 universitários, sendo o gênero feminino predominante (86\%). Ao analisar a prática $42 \%$ dos pesquisados afirmam que se automedicam com certa frequência, sendo que $70 \%$ dos estudantes procuram informações sobre os medicamentos antes de se automedicarem, as informações são obtidas em sua maioria na internet. A classe farmacológica mais utilizada foram as vitaminas, com $95 \%$. Para $45 \%$ a automedicação foi influenciada pelo seu conhecimento sobre os medicamentos por ser estudante da área da saúde. A pesquisa demonstra a importância do tema, promover ações nas instituições de ensino que visam a percepção dos acadêmicos em relação à automedicação e o papel como profissional da saúde a não realização de tal prática.

Palavras-chave: Acadêmicos; Medicamentos; Saúde; Riscos.

\section{Self-medication between universities in the health area inside Tocantins}

The National Health Surveillance Agency (ANVISA) conceptualizes self-medication as indiscriminate use of medications without the prescription, guidance and monitoring of a health professional (doctor or dentist). The act of self-medication among academics in the health field is considered quite common in higher education institutions. It aims to identify the associated factors and the prevalence linked to self-medication in students of a higher education institution in the health area in the interior of Tocantins. This study is a field research, cross-sectional with quantitative analysis, sample selection through randomized means. The research was carried out with Nursing, Biomedicine and Physiotherapy students enrolled at Instituto Educacional Santa Catarina - IESC - Faculdade Guaraí - FAG, in Tocantins, through a virtual questionnaire containing 20 questions. The research is composed by 198 university students, being the predominant female gender ( $86 \%$ ). When analyzing the practice, $42 \%$ of those surveyed state that they self-medicate with a certain frequency, with $70 \%$ of students looking for information about medications before self-medicating, the information is mostly obtained on the internet. The most used pharmacological class was vitamins, with $95 \%$. For $45 \%$, self-medication was influenced by their knowledge about medicines, as they were students in the health field. The research demonstrates the importance of the theme, promoting actions in educational institutions that aim at the perception of academics in relation to self-medication and the role as a health professional of not carrying out such practice.

Keywords: Academics; Medicines; Health; Risks.

Reviewed anonymously in the process of blind peer

Daniele Rodrigues dos Santos Andrade Faculdade Guaraí, Brasil

dannielerodri@gmail.com

Jordana Cardoso dos Santos

Faculdade Guaraí, Brasil

http://lattes.cnpq.br/0944365015455425

jordanasantos196@gmail.com

Giullia Bianca Ferraciolli do Couto

Faculdade Guaraí, Brasil

http://lattes.cnpq.br/0109560699727614

giulliabianca@hotmail.com
Juliane Marcelino dos Santos

Faculdade Guaraí, Brasil

http://lattes.cnpq.br/8751832120632358 julianemarcelino@hotmail.com

Reobbe Aguiar Pereira

Faculdade Guaraí, Brasil

http://lattes.cnpq.br/7447115724350334

enfreobbe@gmail.com

\section{Adriana Keila Dias}

Faculdade Guaraí, Brasil

http://lattes.cnpq.br/2128882976477548

adrianakeiladias@hotmail.com
Glaucya Wanderley Santos Markus

Faculdade Guaraí, Brasil

http://lattes.cnpq.br/5568510365985231

glaucyamarkus@outlook.com

Karla Camila Correia da Silva

Faculdade Guaraí, Brasil

http://lattes.cnpq.br/1981447087125364

karlacamilac@yahoo.com.br

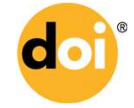

DOI: 10.6008/CBPC2236-9600.2021.003.0014
Referencing this:

ANDRADE, D. R. S.; SANTOS, J. C.; COUTO, G. B. F.; SANTOS, J. M.; PEREIRA, R. A.; DIAS, A. K.; MARKUS, G. W. S.; SILVA, K. C. C.. Automedicação entre universitários da área da saúde no interior do Tocantins. Scire Salutis, v.11, n.3, p.108-117, 2021. DOI: http://doi.org/10.6008/CBPC2236-9600.2021.003.0014 


\section{INTRODUÇÃO}

A Agência Nacional de Vigilância Sanitária (ANVISA) conceitua a automedicação como sendo o uso indiscriminado de medicamentos sem a prescrição, orientação e/ou acompanhamento de um profissional da área da saúde (médico ou dentista) (BRASIL, 2001), a automedicação responsável, por sua vez, é definida pela Organização Mundial da Saúde (OMS) como a prática na qual indivíduos tratam seus próprios sintomas e males menores com medicamentos aprovados para a venda sem a necessidade de prescrição médica, tendo, estes, eficácia e segurança comprovada quando usados seguindo as instruções, e utilizados racionalmente (SÃO PAULO, 2010).

A automedicação é prática consolidada em países como os da União Europeia, nos EUA, Canadá e Japão, sendo a automedicação responsável utilizada, sobretudo no tratamento de sintomas e doenças consideradas sem riscos maiores, como gripes, resfriados, dores de cabeça comuns, entre outras condições clínicas (SÃO PAULO, 2010).

De acordo com Arrais (2016), a população brasileira frequentemente pratica a automedicação, ocorrendo algumas diferenças regionais. Essa prática é predominante no sexo feminino, principalmente em regiões Nordeste, Centro-Oeste e Norte, pela presença de sinais e sintomas que indicam doenças ou condições crônicas e dentre as faixas etárias com maior destaque para todos com idade superior a 18 anos. O autor supracitado mostra ainda que a maioria dos medicamentos utilizados são livres de prescrição, no entanto não são isentos de riscos, com isso não se deve descartar os possíveis efeitos adversos e intoxicações que eles podem ocasionar a seus usuários e ainda contribuem com aumentos com as despesas e serviços de saúde.

O Ministério da Saúde (MS) através da Portaria no 3.916/98 determina a Política Nacional de Medicamentos, que tem como propósito garantir a qualidade dos medicamentos, necessária eficácia, segurança, o incentivo ao seu uso racional e o acesso da população àqueles considerados primordiais (JOÃO, 2010). No entanto, apesar da grande contribuição desta portaria quanto ao uso racional de medicamentos, notou-se uma baixa mudança de hábitos de automedicação por parte da população (SILVA, 2012).

A prática da automedicação entre os acadêmicos da área da saúde é considerada bastante comum nas instituições de ensino superior. Dentre os fatores que contribuem a alta prevalência da automedicação nesse grupo estão nível de confiança, de conhecimento, facilidade de acesso a esses medicamentos, condição financeira, aconselhamento de terceiros, ansiedade em obter alívio rápido, campanhas publicitárias persuasivas, e, em alguns casos, a precariedade dos serviços de saúde. As principais causas que levam os universitários a utilizarem medicamentos sem prescrição são: cefaleias, dores musculares e resfriados (JESUS et al., 2013).

Buscando o alívio imediato de alguns sintomas, a automedicação é vista por muitos como uma solução, gerando consequências mais sérias em alguns casos. Baseado nisso, o presente artigo tem intuito de detectar e mensurar a prática dentro do meio acadêmico. Por ser um grupo que está constantemente envolvido com medicações, estudos de casos clínicos, fisiopatologias e medicações, acaba se tornando 
suscetível por confiar fielmente no conhecimento adquirido no decorrer do curso de graduação, baseandose em diagnósticos sugestivos, indicados por vezes em pesquisas na internet, ignorando totalmente as consequências da automedicação.

Tendo em vista que os acadêmicos da área da saúde serão os futuros educadores de uma população que deverá ser acompanhada e assistida no sistema de saúde e por eles também realizarem uma prática com riscos para o seu bem-estar, emergiu a seguinte questão norteadora: Qual a prevalência e o conhecimento entre acadêmicos da área da saúde acerca dos riscos à saúde associados a prática de automedicação? Diante disso, este artigo tem como principal objetivo identificar a prevalência e os fatores associados à automedicação em estudantes de uma instituição de ensino superior da área da saúde no interior do Tocantins, assim como avaliar o conhecimento dos acadêmicos quanto aos riscos da automedicação, classificar os tipos de fármacos mais utilizados e identificar os motivos que os levam a se automedicarem.

\section{METODOLOGIA}

Este estudo é uma pesquisa de campo, transversal com análise quantitativa, seleção de amostra por meio randomizado. A pesquisa foi realizada com os acadêmicos de Enfermagem, Biomedicina e Fisioterapia matriculados no Instituto Educacional Santa Catarina - IESC - Faculdade Guaraí - FAG, em Guaraí - Tocantins, no segundo semestre de 2020. Utilizou-se o programa Survey Monkey para o cálculo de tamanho de amostra, com um índice de confiança de 95\% e imprecisão de 5\%, desta forma, o tamanho da amostra estimada foi de 200 indivíduos.

Os critérios de inclusão da pesquisa foram acadêmicos que estivessem devidamente matriculados nos cursos de Enfermagem, Biomedicina e Fisioterapia, no segundo semestre de 2020 e aceitassem participar do estudo concordando e assinando o Termo de Consentimento Livre e Esclarecido (TCLE). Foram excluídos desse estudo os que tinham idade abaixo de 18 anos e que recusaram fazer parte do estudo ou não assinar o TCLE. Como ferramenta de coleta de dados foi utilizado um questionário virtual semiestruturado proposto por (LOPES et al., 2017), contendo 20 questões objetivas que abordam riscos e benefícios à saúde em relação ao uso de medicamentos, as classes utilizadas, conhecimentos sobre os fármacos, entre outras. Tal ferramenta auxilia na percepção dos riscos inerentes de fármacos reconhecendo e determinando o estilo de vida dos pesquisados. O instrumento foi aplicado a 198 acadêmicos.

O questionário foi transportado para plataforma online Forms/Microsoft e a divulgação se deu pela postagem de links do questionário em ambientes virtuais utilizados pela população do estudo, como grupos virtuais e e-mails institucionais. Os dados coletados foram dispostos em um banco de dados criado no Microsoft Excel e posteriormente apresentados de forma descritiva, tendo por base tabelas contendo frequências absolutas $(\mathrm{n})$ e relativas (\%). Antecedendo a coleta de dados com o questionário virtual, foi introduzido um Termo de Consentimento Livre e Esclarecido (TCLE) contendo informações e esclarecimentos a respeito da pesquisa, além da solicitação para uso dos dados. 
O progresso do estudo se deu em conformidade com a Resolução № 466, de 12 de outubro de 2012 do Conselho Nacional de Saúde (CNS), onde essa define diretrizes e normas nacionais e internacionais reguladoras para pesquisas que envolvam seres humanos. A pesquisa obteve a aprovação do Comitê de Ética em Pesquisa do Instituto Superior e Centro Educacional Luterano Bom Jesus - IELUSC sob o Parecer № 4.346 .894 .

\section{RESULTADOS E DISCUSSÃO}

A pesquisa foi composta por 198 universitários da área da saúde de uma instituição de ensino superior no interior do Tocantins, sendo que $46 \%$ do curso de Enfermagem, $30 \%$ do curso de Fisioterapia e $24 \%$ do curso de Biomedicina, sendo a maioria (52\%) acima do 60 período. Em relação à faixa etária 35\% dos acadêmicos relataram ter acima de 23 anos, $26 \%$ de 22 a 23 anos, $26 \%$ de 20 a 21 anos e $13 \%$ de 18 a 19 anos. Quanto ao sexo, 86\% dos participantes foram do sexo feminino.

Tabela 1: Distribuição das frequências simples e relativas referente à automedicação dos participantes da pesquisa. Guaraí (TO), Brasil (2020)

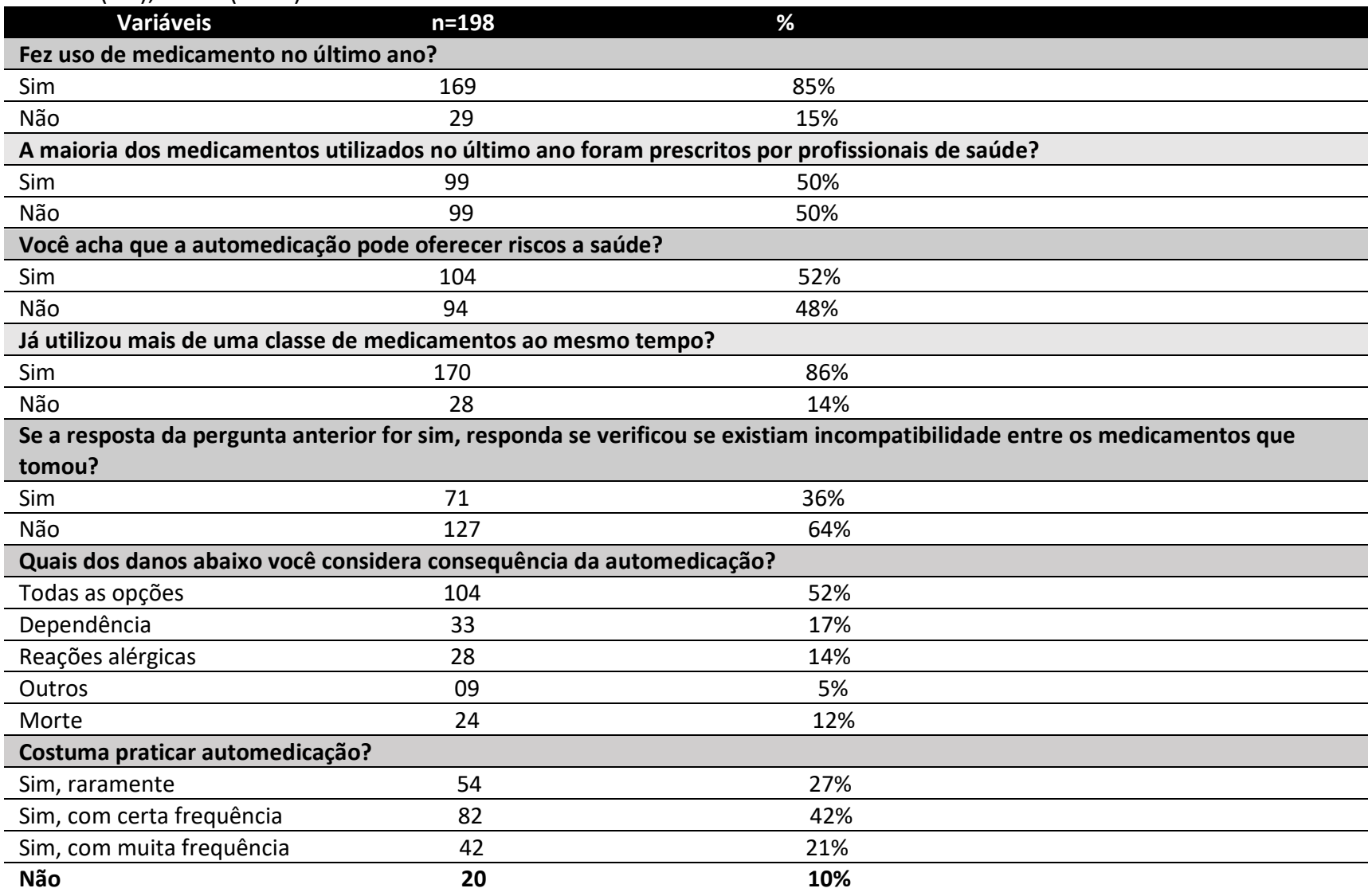

Quando questionados em relação ao uso de medicamentos no último ano, $85 \%$ dos estudantes informaram ter feito uso, sendo que $50 \%$ afirmam que a maioria dos medicamentos utilizados foram prescritos por profissionais da saúde. Segundo dados da pesquisa $52 \%$ da população em estudo acredita que a automedicação pode trazer riscos à saúde.

O estudo demonstrou que $86 \%$ dos pesquisados já utilizaram mais de uma classe de medicamento ao mesmo tempo, e quando perguntados se verificaram incompatibilidade entre os medicamentos $64 \%$ informaram que não e quando questionados sobre quais as possíveis consequências essa prática pode 
causar, $12 \%$ acreditam que a automedicação pode levar à morte, 14\% marcaram reações alérgicas, 17\% dependência e $52 \%$ consideraram todas as opções. Em relação à frequência em que se automedicam $42 \%$ dos pesquisados afirmam que se automedicam com certa frequência, $27 \%$ raramente, $21 \%$ com muita frequência e $10 \%$ afirmam que não se automedicam.

Os resultados corroboram com os dados de Silva et al. (2015), em um estudo realizado na Universidade Estadual de Londrina, onde 72\% relataram consumo de medicamentos nos últimos 15 dias. Resultados estes que são considerados elevados e destacam o alto processo de medicalização da sociedade. Contatou-se no presente estudo, que a maioria dos acadêmicos acreditam que a automedicação traz riscos à saúde. Em um comparativo com o estudo de Moraes et al. (2018), observa-se que 96,58\% dos estudantes tinham consciência dos riscos à saúde pela realização de automedicação. Conforme Domingues et al. (2017), foi evidenciado em seu estudo na área da saúde que 95,7\% dos estudantes reconhecem os riscos pela prática da ação em estudo. Deste modo, pode-se observar que mesmo reconhecendo as implicações negativas da automedicação, esses estudantes continuam em efetuá-la. Tal fato pode ser justificado pelos estudantes se sentirem seguros e confiantes por seu conhecimento adquirido durante o curso de graduação, hipótese esta que é fortalecida pelo achado que aponta que a maioria dos entrevistados já estão em mais da metade da graduação, mais próximos ao término do curso.

Embora a população de estudo tenha demonstrado predominantemente que não ocorreram consequências negativas pela associação de mais de um fármaco, as intoxicações e reações adversas a medicamentos consistem em uma causa importante de mortalidade e admissão hospitalar no Brasil (SANTOS, 2018). O que reforça a relevância do combate ao uso irracional de medicamentos e à prática inapropriada de automedicação com posologia e terapêutica inadequadas. De acordo com o estudo de Lima et al. (2018), quando questionados a frequência com que recorrem à automedicação, 49,51\% afirmaram que realizam essa prática às vezes, 34,80\% raramente, e 15,69\% frequentemente. A pressão do dia a dia em virtude de longas horas de estudo, dores pelo esforço diário nos estudos que podem causar cefaleias e dores musculares, podem estar relacionadas à frequência do uso de medicamentos pelos universitários.

Em um estudo realizado por Coelho et al., (2017), para associar a autopercepção do estado de saúde e a automedicação entre universitários observou-se que os estudantes que se entendiam como doentes praticavam mais a automedicação, o que indica que a autopercepção do estado de saúde pode influenciar as escolhas e práticas de autocuidado. Tal associação denota o valor simbólico do medicamento como mecanismo para restauração da saúde.

No presente estudo observa-se que $70 \%$ dos estudantes procuram informações sobre os medicamentos antes de se automedicarem, as informações são obtidas em sua maioria na internet, conforme $45 \%$ dos pesquisados, em seguida aparece a bula com 30\%, 15\% alegam conseguir informações com farmacêuticos, $7 \%$ com parente ou amigo e 3\% com enfermeiro. No que diz respeito à compreensão quanto as informações recebidas, $82 \%$ afirmaram que sim. Ainda de acordo com a tabela $2,88 \%$ dos participantes da pesquisa descrevem que não surgiu problema de saúde relacionado à medicação ingerida 
na automedicação.

Tabela 2: Distribuição das frequências simples e relativas referente à automedicação dos participantes da pesquisa. Guaraí (TO), Brasil (2020).

\section{$\begin{array}{ll}\text { Variáveis } & \mathbf{N}=198\end{array}$}

Procurou informações sobre o medicamento antes de se automedicar?

\begin{tabular}{lcc}
\hline Sim & 139 & $70 \%$ \\
\hline Não & 59 & $30 \%$ \\
\hline Onde obteve as informações sobre a medicação? & \\
\hline Internet & 78 & $45 \%$ \\
\hline Bula & 53 & $30 \%$ \\
\hline Farmacêutico & 26 & $\mathbf{7} \%$ \\
\hline Parente ou amigo & 12 & $3 \%$ \\
\hline Enfermeiro & 05 & $\mathbf{1 2 \%}$ \\
\hline Surgiu algum problema de saúde relacionado à medicação ingerida na automedicacã ? \\
\hline Sim & $\mathbf{2 4}$ & $\mathbf{8 8 \%}$
\end{tabular}

Cada vez mais a internet tem sido utilizada com a finalidade de se adquirir informações relacionadas à saúde, o problema que os usuários podem encontrar ao utilizá-la como fonte de informação é a validade e a confiabilidade de tais informações, de sua qualidade. É importante que o usuário conheça se os resultados provêm de sites que sejam confiáveis e que tenham tido uma avaliação das informações ali presentes (FOMBELLIDA et al., 2016). De acordo com Santos et al. (2019), a quantidade de informações duvidosas encontradas em diversos sites de saúde é enorme. Dúvidas sobre a efetividade da informação online se dão por falta de controle sobre seu rigor científico e profissional, as consequências relacionadas à influência da internet e o processo de automedicação são negativas.

Apesar de todos os perigos que implicam na busca de informações sobre saúde em fontes duvidosas, é possível perceber que os acadêmicos assumem tal experiência, esta condição manifesta ainda mais a gravidade do problema. Além de efetuarem uma prática potencialmente danosa à saúde, ainda se orientam por fontes inseguras. Sendo assim, é importante ressaltar que os estudantes sejam instruídos ainda na graduação, acerca de se certificarem na busca de informações em meios que se ofereçam qualidade e confiabilidade.

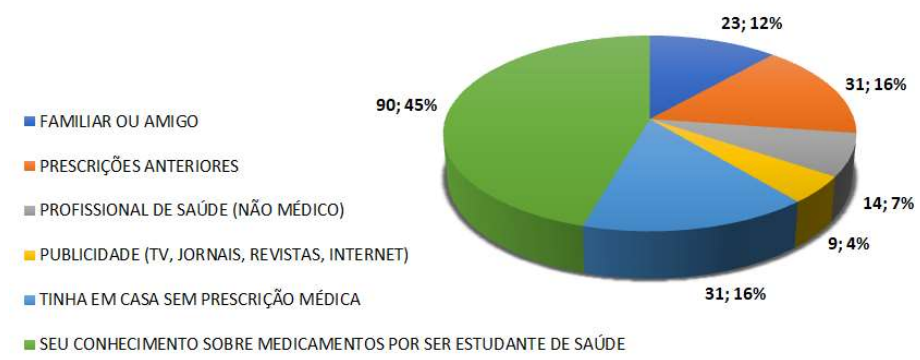

Gráfico 1: O gráfico demonstra possíveis influências que os participantes tiveram em relação a prática automedicação. Guaraí (TO), Brasil (2020).

Conforme dados obtidos no gráfico 01 , para $45 \%$ a automedicação foi influenciada pelo seu conhecimento sobre os medicamentos por ser estudante da área da saúde, $16 \%$ descrevem que foi por prescrições anteriores e porque tinha o medicamento em casa, sem a necessidade de prescrição, $12 \%$ informam que a prática foi por sugestão de familiar ou amigo, 7\% afirmam que foi por profissional de saúde 
(não médico) e, por último, para 5\% dos universitários a automedicação foi influenciada pela publicidade (TV, jornais, revistas e internet).

Ainda de acordo com a pesquisa, $25 \%$ dos discentes afirmam que a prática da automedicação é realizada a partir de experiências anteriores com o medicamento, frente a 19\% discentes que realizam a prática de automedicar-se por julgar os sintomas muito simples, sendo desnecessário ir ao médico. A necessidade de uma melhoria imediata é confirmada por $11 \%$ dos entrevistados. A justificativa de que é fácil e prático ir à farmácia e comprar o medicamento e falta de tempo é admitida por $9 \%$ discentes, $5 \%$ acadêmicos admitem que falta de plano de saúde privado e/ou condição financeira para pagar a consulta e utilização de sobras de medicamentos guardados em casa justificam a prática. A dificuldade em marcação de consultas médicas e compartilhamento de medicamento por alguém de convivência é feita por $4 \%$ discentes. A automedicação influenciada por internet, TV e outros meios de comunicação é admitida por $2 \%$ discentes, assim como a insatisfação em relação ao atendimento médico. A opção outro motivo é confirmado por $7 \%$ dos acadêmicos.

Vários estudos indicam que o grau de conhecimento influencia a prática da automedicação, estando esta atividade intensamente relacionada aos discentes da área da saúde. Sabe-se que os acadêmicos tendem a se automedicar pela autoconfiança, fundamentada no conceito de que eles são possuidores de informações e conhecimentos elevados em relação ao restante da população, em experiências anteriores bem-sucedidas e na confiança de que os conhecimentos alcançados durante a graduação apoiam a seleção adequada do medicamento para determinadas sintomatologias (AQUINO et al., 2010; GALATO et al., 2012; PILGER et al., 2016).

Em relação à influência da automedicação, a pesquisa de Silva et al. (2020) afirma que $74 \%$ dos indivíduos adquiriram os medicamentos em farmácia comercial. Acredita-se que esse seja um fator decisivo para que o Brasil seja um dos países que mais consomem medicamentos no mundo. Ainda de acordo com Silva et al. (2020), acadêmicos da área da saúde tendem a se automedicar e indicar medicamentos para amigos e familiares com maior frequência, por se sentirem aptos e seguros quanto aos efeitos oferecidos. Nos dados coletados na pesquisa supracitada $80,1 \%$ dos indivíduos utilizaram os medicamentos sob influência de amigos, familiares, farmacêuticos, atendentes de farmácia e da mídia. Neste estudo, $85,5 \%$ dos indivíduos entrevistados afirmaram reutilizar os mesmos medicamentos quando apresentam sintomas semelhantes.

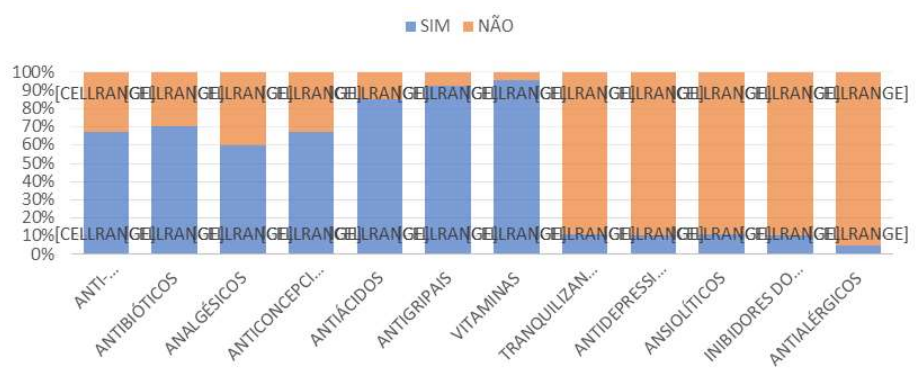

Gráfico 2: 0 gráfico demonstra classes de medicamentos mais utilizados pelos participantes no último ano em relação a prática automedicação. Guaraí (TO), Brasil (2020).

Os resultados apresentados confirmam que a automedicação entre acadêmicos acontece devido a 
confiança no "autodiagnóstico" que são baseados nos sinais e sintomas percebidos pelos entrevistados, despertando uma busca de patologias e tratamentos que se encaixam nos achados. A região que residem os entrevistados também facilita a busca de medicamentos, visto que por ser interior do estado do Tocantins existem vínculos afetivos/parentesco entre moradores e atendentes da saúde e acabam indicando e facilitando a compra de medicações sem prescrição.

Diante dos resultados apresentados no gráfico 02 , o grupo de medicamento mais utilizado sem a prescrição médica foram as vitaminas, sendo utilizada por $95 \%$ discentes, consecutivamente os antigripais por $92 \%$, antiácidos por $85 \%$, antibióticos por $70 \%$, anticoncepcionais e anti-inflamatórios são utilizados por $67 \%$ e analgésicos utilizados por $60 \%$ dos universitários.

O cenário atual de pandemia trouxe consigo a busca irracional por tratamentos. Um estudo realizado no primeiro trimestre de 2020 pela consultoria IQVIA (ROME, 2020), requisitado pelos conselhos regionais de farmácia, corroborou aumento nas vendas de medicamentos (entre eles antigripais, vitaminas e polivitamínicos) que foram ligados à cura, à prevenção e alívio dos sintomas da Covid-19. A automedicação em um momento de colapso sanitário é ativada pela disseminação descompromissada com a ciência. Segundo a OMS (2020), a pandemia pelo Coronavírus veio seguida por uma grande quantidade de informações transportadas nos meios de comunicações sociais sem evidências científicas que bloqueiam o acesso às informações fidedignas a respeito da Covid-19 (PAIVA et al., 2020).

Os elementos coletados confirmam com os dados de Bernardes et al. (2020) em um estudo realizado sobre o perfil epidemiológico de automedicação entre acadêmicos ao longo do curso de medicina na Universidade Federal de Jataí, no qual as categorias de fármacos mais automedicadas entre as classes questionadas estão: anti-inflamatórios, antidepressivos, ansiolíticos e antibióticos. Os anti-inflamatórios, dependendo do intervalo de uso, dosagem e classificação, podem causar sangramento intestinal, úlceras pépticas, síndrome de Reye, levar ao infarto do miocárdio e acidente vascular encefálico em pacientes com insuficiência cardíaca grave e cardiopatia isquêmica. Ainda, em grávidas os anti-inflamatórios não esteroides (AINEs) não são indicados antes da $26^{\circ}$ semanas (fim do segundo trimestre) de gestação. Os próprios estão associados a um histórico de hipersensibilidade manifestada por reações de urticária generalizada, angioedema, edema de glote, hipotensão e choque anafilático.

Segundo Batista (2020), a falta de conscientização da população sobre o uso adequado dos medicamentos e o dimensionamento dos fatores de risco acusados nos tratamentos de enfermidades que não são de origem bacteriana, causa um reflexo no aumento da prevalência da automedicação com antibiótico. De acordo com as entidades de saúde, cerca de 700.000 mil mortes por ano são decorrentes de doenças resistentes aos antimicrobianos. Além disso, constatou-se que antibióticos são utilizados incorretamente, atingindo a resistência e prejudicando o tratamento das doenças.

É perceptível que a prática de automedicação é desregrada, sem acompanhamento de profissionais da área da saúde, sendo realizada em todos os grupos medicamentosos investigados de forma precipitada no meio acadêmico que desconsideram os possíveis riscos do consumo de medicamentos sem prescrição. Essa é realidade do Brasil, no qual a automedicação vem crescendo e tornando-se intensa por vários 
fatores, entre eles a atual pandemia confirmada por vários estudos de diversas regiões do país. É imprescindível que haja fiscalização e atuação dos órgãos competentes frente o uso indiscriminado de medicamentos.

\section{CONCLUSÕES}

Assim, por meio dos dados coletados, foi possível notar que existe uma prática de automedicação por parte dos acadêmicos da área da saúde, além disso, a busca por informações é feita de forma incorreta ou no local inadequado, por exemplo, internet, a maior influência para a automedicação ocorre devido a segurança em ser acadêmico de cursos superiores em saúde, percebe-se também que todas as classes de medicações são utilizadas, algumas em maior percentual que outras.

Após análise e discussão do tema, percebe-se a necessidade de ser abordado o tema automedicação de forma preventiva dentro das Instituições de ensino, por meio de ações educativas que gerem impacto no meio acadêmico, visando assim a conscientização dos acadêmicos em relação à automedicação, os riscos que a sua prática oferecem, como também ressaltando-se a importância da responsabilidade e desenvolvimento de uma visão crítica e consciente em relação a sua ocupação enquanto futuro profissional da saúde.

\section{REFERÊNCIAS}

AQUINO, D.; BARROS, J. A. C.; SILVA, M. D. P.. Selfmedication and health academic staff. Ciencia \& saude coletiva, v.15, n.5, p.2533-2538, 2010.

ARRAIS, P. S. D.. Prevalência da automedicação no Brasil e fatores associados. Revista de Saúde Pública, 2016, DOI: http://dx.doi.org/10.1590/S1518-8787.201605000611

BATISTA, J. A.. Automedicação e Saúde Pública:

Dimensionamento farmacoepidemiológico dos fatores de risco e comportamentos de saúde da população brasileira. Dissertação (Mestrado em Odontologia) - Universidade Estadual Paulista, 2020.

BERNARDES, H. C.. Epidemiological profile of selfmedication among medical academics of a brazilian public university. Monografia (Graduação em Medicina) Universidade Federal de Jataí, Curitiba, 2020.

BRASIL. Agência Nacional de Vigilância Sanitária. Consulta Pública no 95. Agência Nacional de Vigilância Sanitária, 2001.

COELHO, M. T. A. D.. Relação entre a autopercepção do estado de saúde e a automedicação entre estudantes universitários. Revista Psicologia, Diversidade e Saúde, 2017. DOI: http://dx.doi.org/10.17267/23173394rpds.v6i1.1141

MORAES, L. G. M.. Automedicação em acadêmicos de Medicina. Revista da Sociedade Brasileira de Clínica Médica, v.16, n.3, p.167-170, 2018.

DOMINGUES, M. P. S.. Automedicação entre os acadêmicos da área de saúde. Visão Acadêmica, v.18, n.2, 2017. DOI: http://dx.doi.org/10.5380/acd.v18i2.52943
SANTOS, R. C.. A influência da internet no processo de automedicação: uma revisão integrativa/the influence of the internet on the self-medication process: an integrative review. Brazilian Journal of Health Review, v.2, n.5, 2019. DOI: https://doi.org/10.34119/bjhrv2n5-034

JESUS, A. P. G.; YOSHIDA, N. C. P.; FREITAS, J. G.. Prevalência da automedicação entre acadêmicos de farmácia, medicina, enfermagem e odontologia. Revista EVS-Revista de Ciências Ambientais e Saúde, v.40, n.2, p.151-164.

DOI: http://dx.doi.org/10.18224/est.v40i2.2718

JOÃO, W. S. J.. Reflexões sobre o Uso Racional de Medicamentos. Pharmacia Brasileira no 78. 2010.

LIMA, D. M.. Avaliação da prática da automedicação em acadêmicos do curso de Farmácia em uma instituição privada de ensino superior em Fortaleza-CE. Revista Expressão Católica Saúde, v.2, n.1, 2018.

DOI: http://dx.doi.org/10.25191/recs.v2i1.2122

MARTÍN-FOMBELLIDA, A. B.. La información sobre automedicación disponible en internet reúne criterios de calidad?. Revista Cubana de Información en Ciencias de la Salud, v.27, n.1, p.19-34, 2016.

PAIVA, A. M.. Efeito das promessas terapêuticas sobre os preços de medicamentos em tempos de pandemia. Monografia (Graduação em Farmácia) Universidade Federal de São João Del-Rei, Divinópolis, 2020. DOI: http://dx.doi.org/10.12662/23173076jhbs.v8i1.3407.p1-5.2020

PILGER, M. C.; DOMBROWSKI, G. R. M.; TOMASI, E.. Selfmedication among medical students of two medicine schools in Pelotas, South Brazil. Rev AMRIGS, v.60, n.1, 
p.26-31, 2016.

ROME, B. N.; AVORN, J.. Drug Evaluation during the Covid-19 Pandemic. N Engl J Med, v.382, n.24, p.2282-2284, 2020.

SÃO PAULO. Conselho Regional de Farmácia. Medicamentos Isentos de Prescrição. OPAS, 2010.

SANTOS, G. A. S.; BOING, A. C.. Mortalidade e internações hospitalares por intoxicações e reações adversas a medicamentos no Brasil: análise de 2000 a 2014. Cadernos de Saúde Pública, v.34, DOI: https://doi.org/10.1590/0102$\underline{311 \times 00100917}$

SILVA, J. R.; SOUZA, M.; PAIVA, A. S.. Avaliação do uso racional de medicamentos e estoque domiciliar. Ensaios e Ciência: Ciências Biológicas, Agrárias e da Saúde, v.16, n.1, p.109-124, 2012. DOI: https://doi.org/10.17921/1415$6938.2012 \mathrm{v} 16 \mathrm{n} 1 \mathrm{p} \% 25 \mathrm{p}$

SILVA, L. B.. Consumo de medicamentos e prática da automedicação por acadêmicos da área de saúde da Universidade Estadual de Londrina. Espaç. Saúde, p.27-36, 2015. DOI: http://dx.doi.org/10.22421/1517$7130.2015 \mathrm{v} 16 \mathrm{n} 2 \mathrm{p} 27$

SILVA, T. F. Q. E.; GRILLO, L. P.; LACERDA, L. L. V.; MEZADRI, T.. Prevalência de automedicação e características de acesso a anti-inflamatórios em adultos no município de Navegantes, Santa Catarina. Revista Ciências em Saúde, v.10, n.2, p.2027, 2020. DOI: https://doi.org/10.21876/rcshci.v10i2.919

WHO. World Health Organization. Novel Coronavirus (2019nCoV). Genebra: WHO, 2020.

A CBPC - Companhia Brasileira de Produção Científica (CNPJ: 11.221.422/0001-03) detém os direitos materiais desta publicação. Os direitos referem-se à publicação do trabalho em qualquer parte do mundo, incluindo os direitos às renovações, expansões e disseminações da contribuição, bem como outros direitos subsidiários. Todos os trabalhos publicados eletronicamente poderão posteriormente ser publicados em coletâneas impressas sob coordenação da Sustenere Publishing da Companhia Brasileira de Produção Científica e seus parceiros autorizados. Os (as) autores (as) preservam os direitos autorais, mas não têm permissão para a publicação da contribuição em outro meio, impresso ou digital, em português ou em tradução. 